By reviewing the parents' medical history, $23.4 \%$ of mothers and $25.5 \%$ of fathers were diagnosed with either an atopic disease or an inflammatory bowel disease; however, examining the siblings of the affected children, $45,7 \%$ are reported to have one of these conditions (12 children do not have any siblings).

In the study population, $8.5 \%$ were born prematurely and $85.1 \%$ were born at term $(6,4 \%$ did not provide an answer). $46,8 \%$ were exclusively breastfed in the first 6 months of life.

Analysing the data about the forms of clinical presentation, $85.1 \%$ showed gastrointestinal symptoms, $63.8 \%$ demonstrated skin-related problems, $57.4 \%$ presented with respiratory manifestation and $44.7 \%$ showed behavioural problems.

Conclusion According to our results, a positive family history for atopic diseases and/or inflammatory bowel disease can increase the risk of developing cow's milk protein allergy; siblings showed a higher prevalence of food allergy when the parents were affected by the aforementioned entities.

The clinical manifestations represent a wide variety of symptoms which frequently overlap, making the diagnostic process often challenging.

We did not observe a significant effect of feeding (breastfeeding or formula feeding), or being premature on the risk of developing cow's milk protein allergy in children. However, it is important to emphasize the relatively small number of the study population, which is why we plan to continue the research with a larger study group.

\section{P326 EVALUATING THE MANAGEMENT OF IDIOPATHIC CHILDHOOD CONSTIPATION AMONG SECONDARY CARE PAEDIATRICIANS IN WALES}

Davide Paccagnella*, Gareth Davies, Pramodh Vallabhaneni. Department of Paediatrics, Morriston Hospital, Swansea, UK

\subsection{6/archdischild-2019-epa.675}

Background Constipation is a very common paediatric presentation, with an estimated prevalence of $30 \%$ among schoolaged children in the UK. According to NICE guidance, the diagnosis of idiopathic constipation can usually be established by performing a thorough clinical assessment, using amber and red flags to rule out serious underlying causes. Our study is aimed at establishing whether this guideline is adequately used by Paediatricians.

Methods A 10-question survey was sent out to Paediatric Consultants, Specialty doctors, Paediatric trainees and GP trainees working in Paediatrics. The questions were aimed at assessing respondents' knowledge of the NICE recommendations. To contextualise this, clinicians were also asked whether they had experience of and felt confident in treating constipation, and whether specific facilities were available in their department.

Results Data from 23 respondents was analysed. The majority of these (60.9\%) were Paediatric Registrars; 30.4\% were a combination of GP trainees, Paediatric SHOs and Specialty doctors, and $8.7 \%$ were Consultant Paediatricians. Faltering growth was correctly identified as an amber flag by $65.2 \%$ of respondents, whilst only $47.3 \%$ identified possible child maltreatment as one.
All clinicians correctly identified neonatal onset of constipation as a red flag, but only $73.9 \%$ and $52.2 \%$ were able to identify abdominal distention with vomiting and ribbon stools, respectively, as red flags. $39.1 \%$ of respondents stated that they would not routinely investigate idiopathic constipation, whilst up to $56.5 \%$ would perform a coeliac screen. $69.6 \%$ stated they would never perform a digital rectal examination. $43.5 \%$ correctly identified the recommended pathway for treatment and $52.2 \%$ correctly identified the referral criteria. Whilst clinicians felt, on average, moderately confident in managing patients with constipation, up to $56.5 \%$ did not have specific facilities in their department.

Conclusion While most respondents correctly identified amber and red flags, and were reasonably familiar with management recommendations, the majority of clinicians would investigate constipation - which NICE does not routinely recommend. These findings suggest that further targeted education, as well as specialist resources, may improve clinicians' knowledge and confidence in managing this condition and also illustrates guideline fatigue.

\section{P327 SELECTIVE SCREENING FOR CELIAC DISEASE IN CHILDREN WITH ABDOMINAL PAIN}

${ }^{1}$ Valeria Novikova, ${ }^{1}$ Natalia Shapovalova*, ${ }^{1}$ Maria Revnova, ${ }^{2}$ Sergei Lapin, ${ }^{3}$ Elena Kalinina. ${ }^{1}$ St. Petersburg State Pediatric Medical University, St. Petersburg, Russian Federation; ${ }^{2}$ St. Petersburg First Medical University, St. Petersburg, Russian Federation; ${ }^{3}$ Mechnikov I.I. North-Western State Medical University, St. Petersburg, Russian Federation

\subsection{6/archdischild-2019-epa.676}

Introduction Modern epidemiologic data reveal that the prevalence of celiac disease (CD) is approximately $1 \%$ in Europe population. Statistics varies considerably in different countries. The latest Russian clinical recommendations 'Celiac disease in children' published in 2016, suggest the screening for CD only among children with type 1 diabetes mellitus and autoimmune thyroiditis. However, there are no population-based studies and data about prevalence of $\mathrm{CD}$ in average-risk and high-risk groups in Russia.

Aim To determine prevalence of CD in children with abdominal pain.

Methods Tissue transglutaminase antibodies (anti-tTG) of IgA, IgM and IgG classes were detected by quick celiac test «BIOHIT», based on immunochromatographic method. 500 children with abdominal pain, aged $3-17$ years, out patients of the Second diagnostic center in St.Petersburg were examined using the quick-test. All positive for $\mathrm{CD}$ children underwent histological, genetic and serologic tests, as well as in 20 randomly selected children, who didn't test positive. Antibodies to deamidated gliadin peptides (anti-DGPs) and anti-tTG were investigated by enzyme immunoassay (ELISA). The study patients were genotyped for HLA-DQB1and DQA1 alleles using realtime polymerase chain reaction.

Results The test detected 6 positive results $(5$ females with an average age of $12 \pm 2,1$ years and one 10 years old boy) in group with abdominal pain. CD in all this subjects was diagnosed and confirm for first time ever. Quick celiac test «BIOHIT», yield sensitivity 95\% and specificity $100 \%$

Conclusion The prevalence of CD in children with abdominal pain was 1: 83. Quick celiac test is suitable for screening CD in population-based studies, as well as for the diagnosis of this disease in risk groups. 